\title{
The cerebellum on the rise in human emotion
}

\author{
DENNIS J. L. G. SCHUTTER \& JACK VAN HONK \\ Affective Neuroscience Section, Department of Psychonomics, Helmholtz Research Institute, Utrecht University, Utrecht, \\ The Netherlands
}

\begin{abstract}
For decennia the cerebellum has largely been excluded from scientific enquiry beyond motor function. However, the intimate afferent and efferent connections to the midbrain and limbic system provide for the neuroanatomical foundation of cerebellar involvement in emotion and emotional disorders. Moreover, an increasing body of empirical evidence indicates that the cerebellum may be involved in emotion regulation. Both functional and structural abnormalities of the cerebellum have been demonstrated in emotional disorders, including depression and schizophrenia. Research shows that the functional repertoire of the cerebellum is broader than previously thought and its involvement in emotion is noteworthy.
\end{abstract}

Key words: Cerebellum, clinical, emotion, homeostasis, motivation, neuroscience, pacemaker, psychopathology, universal cerebellar transform

\section{Introduction}

The cerebellum is located in the posterior fossa and occupies approximately $10 \%$ of total intracranial volume and contains more than half of the total number of neurons in the human brain (1). The cerebellum is comprised of a three-layered cortex, white matter and cerebellar nuclei $(\mathrm{CN})$. The outermost layer is made up of input stellate and basket cells (lamina molecularis), and the intermediate layer is composed of output inhibitory Purkinje cells (PC). The most internal layer consists of input granule and Golgi cells (lamina granularis). The DCN consist of the dentate nucleus, nucleus emboliformus, globose nucleus and fastigial nucleus, which distribute PC input to non-cerebellar brain structures. Information is conveyed back into the lamina molecularis and granularis of the cerebellar cortex by excitatory afferent input through climbing and mossy fibres that mainly originate from the inferior olive and pontine nucleus respectively. The cerebellum is known for its unique folial surface and well-established involvement in motor function. Traditionally, the cerebellum has not received much attention from researchers studying non-motoric aspects of human behaviour, such as cognition and emotion. Nevertheless some scientists in the late 1970s acknowledged the importance of the cerebellum in emotion and emotional disorders $(2,3)$. It was however the innovative work of researchers such as Jeremy
Schmahmann that extended the functional role of the cerebellum into the domain of cognition and emotion (4).

Further support for the cerebellum-emotion link was provided by clinical studies that yielded strong evidence for cerebellar abnormalities in emotional disorders, including schizophrenia and depression (5). Table I depicts an overview of important contributions from different lines of research illustrating the role of the cerebellum in emotion and emotional disorders. However, one of the more difficult issues that needs to be tackled in future research relates to the specific role of the cerebellum in emotion. Is the cerebellum, for example, part of the brain circuitry involved in the regulation of affective processes or does it merely function as a relay station in the loops connecting cortical and subcortical regions?

Motivation and emotion represent facets of a common core phenomenon, the motivationalemotional system, that interacts with general purpose processing systems of conditioning, learning and higher order cognition (6). Motivation can be defined as the internal drive of an organism, whereas emotions can be considered as the readout of this internal drive when activated by a challenging stimulus $(6,7)$.

The intimate afferent and efferent connection to the brainstem and limbic system are a neuroanatomical foundation for cerebellar involvement in

Correspondence: Dennis J. L. G. Schutter, Helmholtz Research Institute, Affective Neuroscience Section, Utrecht University, Heidelberglaan 2 , 3584 CS Utrecht, The Netherlands. E-mail: D.Schutter@fss.uu.nl 
Table I. Brain research showing the involvement of the cerebellum in emotion and emotional disorders.

\begin{tabular}{|c|c|c|}
\hline Research domain & Authors (Refs) & Main finding \\
\hline \multirow[t]{4}{*}{ Functional neuroanatomy } & Heath et al. $(3,7)$ & $\begin{array}{l}\text { Limbic system responses and mood improvement to electric } \\
\text { stimulation of cerebellum }\end{array}$ \\
\hline & Supple et al. (8) & Relationship between vermis and fear learning \\
\hline & Schutter et al. (37) & $\begin{array}{l}\text { Transcranial magnetic stimulation over cerebellum induces positive } \\
\text { affect }\end{array}$ \\
\hline & Sachetti et al. (9) & $\begin{array}{l}\text { Induction of long term potentiation of the cerebellum by fear } \\
\text { conditioning }\end{array}$ \\
\hline \multirow[t]{3}{*}{ Brain damage } & Schmahmann \& Sherman (15) & $\begin{array}{l}\text { Posterior fossa lesions: CCAS; Behavioural disinhibition, blunting } \\
\text { of affect, psychosis and cognitive dysmetria }\end{array}$ \\
\hline & Levisohn et al. (16) & $\begin{array}{l}\text { CCAS after cerebellar tumour resection in children: Vermal damage } \\
\text { resulted in dysregulation of affect }\end{array}$ \\
\hline & Duggal (17) & $\begin{array}{l}\text { Olivopontocerebellar atrophy: Behavioural disinhibition, sadness, fear } \\
\text { and auditory hallucinations }\end{array}$ \\
\hline \multirow[t]{3}{*}{ Functional neuroimaging } & Liotti et al. (10) & Cerebellum activity during sadness and anxiety in healthy volunteers \\
\hline & Habel et al. (12) & Cerebellum activity during positive mood states \\
\hline & Wiech et al. (11) & $\begin{array}{l}\text { Positive correlation between cerebellum activity and experience of } \\
\text { chronic pain }\end{array}$ \\
\hline \multirow[t]{9}{*}{ Psychiatry } & Ichimiya et al. (26) & Reduced volumes of vermis in neuroleptic naïve schizophrenic patients \\
\hline & Loeber et al. (24) & Reduced volumes of inferior cerebellum in schizophrenia \\
\hline & Kyosseva (23) & Cerebellar abnormalities in schizophrenic patients \\
\hline & Okugawa et al. (21) & $\begin{array}{l}\text { White matter abnormalities in the middle cerebellar peduncles } \\
\text { of schizophrenic patients }\end{array}$ \\
\hline & Okugawa et al. (25) & Smaller volumes of vermis in chronic schizophrenia \\
\hline & Soares \& Mann (27) & Depression linked to reduced volumes of the cerebellum \\
\hline & Beyer et al. (29) & Volume reductions of vermis associated with depression \\
\hline & Leroi et al. (28) & Degenerative cerebellar diseases associated with depression \\
\hline & Neil et al. (30) & Vermal volume reductions in manic-depressive disorder \\
\hline
\end{tabular}

CCAS: Cerebellar Cognitive Affective Syndrome.

emotion and emotional disorders. The cerebellum has not only mono-synaptic projections to the hypothalamus, septum, hippocampus, amygdala and basal ganglia, but cerebellar projections also extend to brainstem nuclei where they stimulate dopamine and noradrenalin release by innervating the substantia nigra and locus coeruleus. Earlier animal studies already showed reduced aggressive behaviour after isolated lesions of the cerebellum in monkeys (8). Furthermore, neuronal responses in limbic brain structures, including septum, hippocampus and amygdala were observed after electrically stimulating the cerebellum of cats and rats (9). Decisive evidence for cerebellar involvement in human emotion was provided by Heath's pacemaker studies that showed positive effects on mood in mentally disturbed patients who received electric cerebellar stimulation via subdurally implanted electrodes (3).

A link between the cerebellum and fear was demonstrated during a Pavlovian fear conditioning study in which extra-cellular single-unit responses of Purkinje cells in the anterior cerebellar vermis varied as a function of learning (10). Additionally, the induction of long-term potentiation in the cerebellum to cued fear conditioning provides corroborating evidence for involvement in fear learning (11). Furthermore, a human functional neuroimaging study in healthy volunteers showed that cerebellar activity was associated with anxiety and sad mood (12). Moreover, involvement of the cerebellum in negative affective states was again evidenced by a relationship between tonic pain and neuronal activity in regions of the medial prefrontal cortex and cerebellum. Interestingly, both the activity in the cerebellum and prefrontal activity decreased as a function of cognitive workload, which was interpreted as an integrative process of motivational and cognitive factors in relation to physical injury and coping (13). The argument for a specific role of the cerebellum in the experience of negative emotional states has however been compromised by a recent study that showed cerebellar activation after the induction of positive mood in healthy male subjects (14). Although both positive and negative mood were associated with common activity in several cortical and limbic structures, cerebellar activity seemed to relate to positive, but not negative mood. In sum, the relationship between the cerebellum and emotion finds increasing support, but whether or not the cerebellum is specialized in particular forms of emotional processes remains an open question.

The involvement of the cerebellum in motivation and emotion may at least partially be established through its interactions with the endocrine system. In particular, the cerebellar-hypothalamic connection yields an important neuroanatomical pathway 
for humoral interaction. A recently discovered cerebellar peptide, called cerebellin seems to stimulate the hypothalamic-pituitary-adrenal (HPA) axis, as evidenced by increases in its end-product cortisol (15), the major stress hormone that is associated with both anxiety and depression. The reciprocal relationship between the cerebellum and HPA-axis was recently established by the discovery of dense glucocorticoid binding sites on the vermis (16).

In addition to the functional neuroanatomical evidence, lesion studies provide a valuable source for investigating the relationship between the cerebellum and emotion. A syndrome resulting from cerebellar damage has been termed the cerebellar cognitive-affective syndrome (CCAS) and involves both emotional and cognitive impairments (17). Executive dysfunction, behavioural disinhibition and blunting of affect are among the characteristic signs and symptoms associated with particularly posterior and vermal lesions (17).

This notion is supported by a paediatric study that showed CCAS in children who had undergone cerebellar tumour resection. In particular, damage to the vermis has been associated with disturbances in emotion regulation (18). Interestingly, the CCAS shares many commonalities with the classical frontal lobe syndrome, which is demonstrated by a patient with sporadic olivopontocerebellar atrophy who displayed behavioural disinhibition and unprovoked laughter (19), disturbances that are not uncommon in patients with frontal damage.

Furthermore, cerebellar abnormalities have also been observed in schizophrenia, a 'dysconnectivity' syndrome characterized by uncoordinated affective and cognitive processing, collectively termed cognitive dysmetria (20). Anatomical evidence for disruptions of neural connectivity was provided by a diffusion tensor imaging (DTI) study showing white matter abnormalities in the middle cerebellar peduncles of schizophrenic patients (21). Parallel to the traditional role of the cerebellum in the modulation of motor activity, it has been argued that the cerebellum also holds a central position in the regulation of mental activity. Although no convincing evidence for cerebellar abnormalities were found for adolescent-onset schizophrenia (22), recent post-mortem and neuroimaging studies do indicate cerebellar abnormalities in schizophrenia (23). Again the vermis seems to be involved; several structural neuroimaging studies in schizophrenia have provided evidence for distinct volume reductions involving the vermis, but not the cerebellar hemispheres $(24,25)$. Deleterious effects of antipsychotic medication on brain morphology cannot be ruled out in this research, but a study involving 20 neuroleptic-naïve schizophrenic males also showed reductions of the cerebellar vermis (26). The dysregulation of emotion has also been argued to be involved in several other forms of psychopathology.
For example, depression has not only been associated with volumetric reductions of the frontal lobes, but also of the cerebellum (27). Further evidence for the relationship between the cerebellum and depression was demonstrated in degenerative cerebellar disease. In a patient study, comprehensive psychiatric assessment revealed that depression was associated with cerebellar degeneration (28). This relationship was recently confirmed by a systematic review of the literature that indicated abnormalities in the frontal lobes, basal ganglia and cerebellum (29). It is probably no coincidence that depression is often associated with psychomotor disturbances involving gait, posture and coordination of movements, disturbances which are typically found in cerebellar ataxia. Finally, evidence is now accumulating that vermal abnormalities are also present in bipolar disorders (30).

The cerebellum might hold a central position and act as a convergence zone for the different information streams. Indeed, the highly topographically organized information flow from and to the cerebellum in so-called micro-complexes suggests that the cerebellum plays an important role in the regulation of adaptive emotional and motivational behaviour. The morphological uniformity of the cerebellar cortex and the concept of a cerebellar regulatory system has been deemed the universal cerebellar transform (UCT) hypothesis (31). According to the UCT hypothesis the cerebellum acts as a pacemaker establishing and maintaining organism's behaviour at an optimum level. The notion that the cerebellum is equipped with internal and external representations, which provide a neuronal foundation for monitoring and integrating intero- and exteroceptive information is substantiated by findings of cerebellar involvement in error monitoring (32). In this sense, the function of the cerebellum is analogous to a supramodal internal timing unit. Hypothetically, when the cerebellar timing function is disrupted, information processing streams become desynchronized, providing a breeding ground for a diverse range of psychopathological conditions. Recent studies have established a reciprocal anatomical pathway between the cerebellum and prefrontal cortex (33), and thus the neocortical area is importantly involved in emotion and emotional disorders. The cerebellar connections to the brainstem, limbic system and prefrontal cortex in the UCT model (31) predict an important role for the cerebellum in emotion processing. However, at present the exact functional contribution of the cerebellum in emotion remains unclear. A possible way of gathering insights into the functional role of the human cerebellum in the regulation of emotion may be provided by transcranial magnetic stimulation (TMS). TMS can be considered as the modern non-invasive analogue to Heath's electric cerebellar stimulation technique. TMS is a simple, though 
ingenious method of passing electric currents through the scalp into the brain by means of magnetic fields (34) and can be utilized to investigate brain function more directly by applying brief magnetic pulses to either disrupt of facilitate cortical information processing (35). An electric current is conveyed into the brain through a brief, but strong magnetic field. Near nerve cells the magnetic field is subsequently transformed back into an electric current causing a transmembrane potential, which induces neuronal depolarization and an action potential. Interestingly, in a recent patient study TMS was applied over the cerebellum and support was provided for dysfunctional cerebello-cortical connectivity in schizophrenia (36). Furthermore, in another study it was shown that twenty minutes of cerebellar TMS in healthy volunteers modified neuroelectric recordings over the prefrontal cortex and increased positive mood and alertness (37). These data concur with animal studies wherein activation of the mesencephalic reticular formation through cerebellar stimulation resulting in cortical arousal and alertness (38). In sum, the exploration of cerebellar TMS in the treatment of emotional disorders may well be worthwhile (39).

The involvement of the cerebellum in affective processing was until recently neglected, but currently there is a flourishing interest in studying the link between the cerebellum and emotion. An important role in the regulation of emotion seems reserved for the cerebellar vermis. It has even been suggested that the vermis together with the fastigial nucleus and flocculonodular lobe are an extension of Papez's emotion circuit; the limbic cerebellum $(1,30)$. Further research is however needed to establish the exact role of the cerebellum in 'normal' and pathological forms of emotion processing.

\section{Acknowledgements}

Dr Schutter was supported by an Innovational Research Grant (\# 451-04-070) from the Netherlands Organization for Scientific Research (NWO). Dr Van Honk was supported by an Innovational Research Grant (\# 016-005-060) from the Netherlands Organization for Scientific Research (NWO).

\section{References}

1. Kandel ER, Schwartz JH, Jessel TM. Principles of neural science (4th edn). New York: McGraw-Hill; 2000.

2. Snider RS, Maiti A. Cerebellar contributions to the Papez circuit. J Neurosci Res. 1976;2:133-46.

3. Heath RG. Modulation of emotion with a brain pacemaker: Treatment for intractable psychiatric illness. J Nerv Ment Dis. 1977;165:300-17.

4. Schmahmann JD. An emerging concept. The cerebellar contribution to higher function. Arch Neurol. 1991;48: $1178-87$.
5. Schmahmann JD. Disorders of the cerebellum: Ataxia, dysmetria of thought, and the cerebellar cognitive affective syndrome. J Neuropsychiatry Clin Neurosci. 2004;16: 367-78.

6. Buck R. The biological affects: A typology. Psychol Rev. 1999;106:301-36.

7. Papez JW. A proposed mechanism of emotion. Arch Neurol Psychiatry. 1937;38:725-43.

8. Peters M, Monjan AA. Behavior after cerebellar lesions in cats and monkeys. Physiol Behav. 1971;6:205-6.

9. Heath RG, Dempesy CW, Fontana CJ, Myers WA. Cerebellar stimulation: Effects on septal region, hippocampus, and amygdala of cats and rats. Biol Psychiatry. 1978; 13:501-29.

10. Supple WF Jr, Sebastiani L, Kapp BS. Purkinje cell responses in the anterior cerebellar vermis during Pavlovian fear conditioning in the rabbit. Neuroreport. 1993;4:975-8.

11. Sacchetti B, Scelfo B, Tempia F, Strata P. Long-term synaptic changes induced in the cerebellar cortex by fear conditioning. Neuron. 2004;42:973-82.

12. Liotti M, Mayberg HS, Brannan SK, McGinnis S, Jerabek P, Fox PT. Differential limbic-cortical correlates of sadness and anxiety in healthy subjects: Implications for affective disorders. Biol Psychiatry. 2000;48:30-42.

13. Wiech K, Seymour B, Kalisch R, Enno Stephan K, Koltzenburg M, Driver J, Dolan RJ. Modulation of pain processing in hyperalgesia by cognitive demand. Neuroimage. 2005;27:59-69.

14. Habel U, Klein M, Kellermann T, Shah NJ, Schneider F. Same or different? Neural correlates of happy and sad mood in healthy males. Neuroimage. 2005;26:206-14.

15. Mazzocchi G, Andreis PG, De Caro R, Aragona F, Gottardo L, Nussdorfer GG. Cerebellin enhances in vitro secretory activity of human adrenal gland. J Clin Endocrinol Metab. 1999;84:632-5.

16. Teicher $\mathrm{MH}$, Andersen SL, Polcari A, Anderson CM, Navalta CP, Kim DM. The neurobiological consequences of early stress and childhood maltreatment. Neurosci Biobehav Rev. 2003;27:33-44.

17. Schmahmann JD, Sherman JC. The cerebellar cognitive affective syndrome. Brain. 1998;121:561-79.

18. Levisohn L, Cronin-Golomb A, Schmahmann JD. Neuropsychological consequences of cerebellar tumour resection in children: Cerebellar cognitive affective syndrome in a paediatric population. Brain. 2000;123:1041-50.

19. Duggal HS. Cognitive affective psychosis syndrome in a patient with sporadic olivopontocerebellar atrophy. J Neuropsychiatry Clin Neurosci. 2005;17:260-2.

20. Andreasen NC. A unitary model of schizophrenia. Arch Gen Psychiatry. 1999;56:781-7.

21. Okugawa G, Nobuhara $K$, Sugimoto $T$, Kinoshita $T$. Diffusion tensor imaging study of the middle cerebellar peduncles in patients with schizophrenia. Cerebellum. 2005;4:123-7.

22. James AC, James S, Smith DM, Javaloyes A. Cerebellar, prefrontal cortex, and thalamic volumes over two time points in adolescent-onset schizophrenia. Am J Psychiatry. 2004;161:1023-9.

23. Kyosseva SV. The role of the extracellular signal-regulated kinase pathway in cerebellar abnormalities in schizophrenia. Cerebellum. 2004;3:94-9.

24. Loeber RT, Cintron CM, Yurgelun-Todd DA. Morphometry of individual cerebellar lobules in schizophrenia. Am J Psychiatry. 2001;158:952-4.

25. Okugawa G, Sedvall GC, Agartz I. Smaller cerebellar vermis but not hemisphere volumes in patients with chronic schizophrenia. Am J Psychiatry. 2003;160:1614-17.

26. Ichimiya T, Okubo Y, Suhara T, Sudo Y. Reduced volume of the cerebellar vermis in neuroleptic-naive schizophrenia. Biol Psychiatry. 2001;49:20-7. 
27. Soares JC, Mann JJ. The anatomy of mood disorders-review of structural neuroimaging studies. Biol Psychiatry. 1997; 41:86-106.

28. Leroi I, O’Hearn E, Marsh L, Lyketsos CG, Rosenblatt A, Ross CA, Brandt J, Margolis RL. Psychopathology in patients with degenerative cerebellar diseases: A comparison to Huntington's disease. Am J Psychiatry. 2002;159: 1306-14.

29. Beyer JL, Krishnan KR. Volumetric brain imaging findings in mood disorders. Bipolar Disord. 2002;4:89-104.

30. Neil P, Mills NP, DelBello MP, Caleb M, Adler CM, Strakowski SM. MRI analysis of cerebellar vermal abnormalities in bipolar disorder. Am J Psychiatry. 2005; 162:1530-3.

31. Schmahmann JD. The role of the cerebellum on affect and psychosis. J Neurolinguist. 2000;13:189-214.

32. Schweighofer N, Doya K, Kuroda S. Cerebellar aminergic neuromodulation: Towards a functional understanding. Brain Res Rev. 2004;44:103-16.

33. Middleton FA, Strick PL. Cerebellar projections to the prefrontal cortex of the primate. J Neurosci. 2001;21: 700-12.
34. George MS, Belmaker RH. Transcranial magnetic stimulation in neuropsychiatry. Washington, DC: American Psychiatric Press; 2000.

35. Grafman J, Wassermann EM. Transcranial magnetic stimulation can measure and modulate learning and memory. Neuropsychologia. 1999;37:159-67.

36. Daskalakis ZJ, Christensen BK, Fitzgerald PB, Fountain SI, Chen R. Reduced cerebellar inhibition in schizophrenia: a preliminary study. Am J Psychiatry. 2005;162:1203-5.

37. Schutter DJLG, van Honk J, d'Alfonso AAL, Peper JS, Panksepp J. High frequency repetitive transcranial magnetic over the medial cerebellum induces a shift in the prefrontal electroencephalography gamma spectrum: a pilot study in humans. Neurosci Lett. 2003;336:73-6.

38. Schmahmann JD, Anderson CM, Newton N, Ellis R. The function of the cerebellum in cognition, affect and consciousness: Empirical support for the embodied mind. Conscious Emotion. 2001;2:273-309.

39. Schutter DJLG, Van Honk J. A framework for targeting alternative brain regions with repetitive transcranial magnetic stimulation in the treatment of depression. J Psychiatry Neurosci. 2005;30:91-7. 